\title{
Optical coherence tomography in coronary atherosclerosis
}

\begin{abstract}
Despite developments in therapeutic and diagnostic technologies, the global burden of atherosclerotic coronary artery disease is increasing. Intravascular imaging has become an invaluable adjunct to percutaneous coronary intervention. Optical coherence tomography (OCT) is a catheter based invasive imaging system that uses light instead of ultrasound to produce high resolution in vivo images of coronary arteries and deployed stents. The technical aspects of intracoronary image acquisition, advantages, and disadvantages of use and current applications of OCT in coronary atherosclerosis are discussed.
\end{abstract}

Keywords: optical coherence tomography, atherosclerosis, coronary artery disease

\author{
Volume I Issue I - 2015 \\ Nikunj R Shah,,'2 Philippa J Howlett,' David \\ S Fluck, ${ }^{2}$ Mark Williams, ${ }^{2}$ Adam Jacques, ${ }^{2}$ \\ Michael Mahmoudi, 1,2 \\ 'Faculty of Health and Medical Sciences, University of Surrey, \\ UK \\ ${ }^{2}$ Ashford \& St. Peter's Hospital NHS Foundation Trust, UK
}

Correspondence: Nikunj R Shah, Faculty of Health and Medical Sciences, University of Surrey, UK, Tel 07974 56I I 97, Email nikunjravshah@hotmail.com

Received: July 09, 2015 | Published: July 23, 2015
Abbreviations: IVUS, intravascular ultrasound; OCT, optical coherence tomography; FFR, fractional flow reserve; TD-OCT, tissue-domain optical coherence tomography; FD-OCT, frequencydomain optical coherence tomography; PCI, percutaneous coronary intervention; TCFA, thin-capped fibroatheroma; MACE, major adverse cardiovascular event

\section{Introduction}

Despite advances in therapeutic and diagnostic technologies, ischaemic heart disease remains a global leading cause of death. Most cases result from atherosclerosis, an inflammatory and fibroproliferative process resulting in activation of growth factors, vasoregulatory mechanisms and cytokines resulting in intimal thickening and subsequent endoluminal obstruction. ${ }^{2}$ More sophisticated interventional techniques have been adopted to combat the increasing burden of atherosclerosis, including intravascular ultrasound (IVUS), fractional flow reserve (FFR) and more recently, optical coherence tomography (OCT). This paper provides an overview of the principles and current uses of OCT in diagnosing and guiding the treatment of atherosclerotic coronary artery disease.

\section{Principles of optical coherence tomography}

Intravascular OCT provides a method of obtaining cross-sectional tomographic vascular imaging with definition that is superior to other currently available modalities. It acts as an optical analogue of IVUS, where ultrasound is replaced by light that is reflected or back-scattered from internal structures within tissue. This 'echo time delay' produces a measurable signal intensity or magnitude. As the speed of light does not allow direct measurement of the echo time delay, interferometric techniques are employed to analyse the reflected light signal. Two main technologies exist to create OCT images, time domain (TDOCT) and Fourier or frequency domain (FD-OCT). While TD-OCT uses a moving mirror as its reference arm and a broadband light source, FD-OCT uses a fixed mirror with a variable frequency light source allowing simultaneous detection of reflections from all echo time delays. ${ }^{3}$ This allows faster image acquisition rates, improved signal-to-noise ratio with subsequent higher quality imaging, making FD-OCT the imaging mode of preference. ${ }^{4}$ By utilising this ultrafast frequency swept near-infrared light source to image the vessel, FDOCT is able to gain axial resolutions in the region of 10 to $15 \mu \mathrm{m}$ and lateral resolutions of 20 to $90 \mu \mathrm{m}$. The rotating fibre-optic system creates a detailed tissue image with a ten-fold greater resolution than that achieved with IVUS, ${ }^{5-7}$ due to the shorter wavelength (1280$1350 \mathrm{~nm}$ range) of the imaging light compared with ultrasound. However, the shorter wavelength limits tissue penetration to 1 to $3 \mathrm{~mm}$ as compared with 4 to $8 \mathrm{~mm}$ achieved with IVUS, with the exception of calcified lesions in which ultrasound has a limited penetration. ${ }^{8}$

Due to the high attenuation of light by red blood cells, effective OCT requires the clearing or flushing of blood from the lumen prior to imaging the desired segment of vessel. The slow acquisition speed of TD-OCT requires proximal balloon occlusion of the coronary of interest, with subsequent flushing of the artery to remove blood from the field of view. This limits its widespread adoption because of prolonged intravascular occlusion potentiating the risk of coronary damage and myocardial ischaemia. In FD-OCT, a greater frame rate is achieved because the swept source laser can be focussed quicker than the reference mirror can be moved in TD-OCT. Therefore, comprehensive volumetric microscopy of the vessel can be safely performed with the intra-arterial flushing of blood with a $10-15 \mathrm{ml}$ bolus of crystalloid solution, usually radio contrast, obviating the need for balloon occlusion of the vessel. ${ }^{9}$

\section{OCT in coronary atherosclerosis, percutaneous coronary intervention and plaque characterisation}

Both TD and FD-OCT are deemed safe in assessing atherosclerotic plaque characteristics, as well as guiding percutaneous coronary intervention (PCI). ${ }^{10-13}$ The high definition of OCT imaging allows all aspects of plaque morphology to be accurately delineated including fibrous tissue, lipid accumulation and calcific deposition. ${ }^{6,14}$

A large proportion of acute coronary events arise from sudden luminal obstruction caused by thrombosis as a result of plaque rupture or erosion. Our current understanding of vulnerable plaque biology suggests that approximately $80 \%$ of significant plaque rupture occurs within inflamed thin-capped fibroatheromas (TCFA).$^{15}$ It is accepted that TCFA comprise of a thin fibrous cap less than $65 \mu \mathrm{m}$ in diameter, 
overlying a large necrotic lipid pool ( $>2$ quadrants of the crosssectional image), with associated inflammatory cell cap infiltration. ${ }^{16}$ Studies using virtual histology IVUS have previously determined TCFA as a predictor of major adverse cardiovascular events (MACE). ${ }^{17,18}$ As OCT provides superior in vivo characterisation of plaque morphology, identifying TCFA may prospectively identify the most vulnerable lesions and therefore influence prognosis. This has been validated in clinical studies comparing OCT with IVUS in acute coronary cohorts, ${ }^{16,19}$ particularly as a rupture-prone fibrous cap less than 65 microns is well under the resolution capacity of IVUS. In vivo and post mortem studies have also corroborated the ability of OCT to accurately detect TCFA, especially in identifying its increased incidence in myocardial infarction. ${ }^{20-22}$ Furthermore, OCT has been successfully used to examine the role of pharmacological interventions on plaque anatomy and stability. ${ }^{23-25}$

In normal vessels, the coronary artery appears as a three layered structure with OCT. A dark band denotes the low signal muscular media layer between the internal elastic lamina and external elastic lamina. Due to its limited tissue penetration, however, OCT is not well suited to assess significant vascular remodelling and total plaque volume. Fibrous plaques tend to appear as high signal homogenous regions with high levels of back-scatter. Calcifications within plaques are identified by the presence of well circumscribed, low back-scattering heterogenous areas. , $^{6,8,14,19}$ Lipid pools, in contrast, are less well delineated than calcification and appear as diffusely bordered, low signal areas with more heterogenous back-scattering than fibrous plaques. ${ }^{6,8,14,19}$ In reality, an overlying signal-rich band usually corresponds to a fibrous cap of varying thickness (Figure 1). Thrombi are identified by the protrusion of a mass into the vessel lumen discontinuous from the arterial wall and may be found in culprit lesions of patients with acute coronary syndromes. ${ }^{6,8,14,19,26,27}$ In the early stages of thrombogenesis, a rapidly organising thrombus traps blood cell constituents and on maturation, it becomes more fibrous and less cellular, turning from 'red' to 'white'. OCT has been shown to accurately distinguish between red and white thrombi, and may therefore aid in determining optimal therapeutic strategy. ${ }^{26}$ Red thrombi, consisting mainly of signal attenuating red cells, are characterised by high back-scattering protrusions with signal-free shadowing. White thrombi, comprising predominantly of platelets and white cells, appear as signal-rich, low back-scattering projections into the vessel lumen. ${ }^{26}$ Plaque ulceration or rupture can be detected by OCT as a ruptured fibrous cap that connects the lumen with the lipid pool. These may or may not be associated with superimposed thrombus Table 1 .

In addition to plaque characterisation, OCT has been used to optimise and guide PCI by providing a clear depiction of the boundaries between vessel, lumen and metallic stent struts. The near infra-red OCT light does not penetrate metal, hence stent struts are visualized as linear structures with strong surface reflection and typical dorsal shadowing. ${ }^{28}$ Earlier papers allude to OCT outperforming IVUS in detecting spontaneous and PCI-induced dissection, tissue prolapse and incomplete stent apposition, all of which have been implicated in acute as well as late stent thrombosis. ${ }^{12,29,30}$ Limited outcome data also exists to show OCT-guided PCI as superior to angiography alone. ${ }^{31}$ This unrivalled endovascular resolution has allowed assessment of the vascular response at individual stent strut level following device deployment. Hence, numerous in-vivo OCT studies have exploited its capability to study factors related to the prognosis of stent-implanted lesions, including neointimal hyperplasia, stent strut coverage, stent malapposition and in-stent neoatherosclerosis. ${ }^{32-34}$ Technical drawbacks to OCT, in addition to limited tissue depth penetration, include inability to image plaques located at the very ostium of the coronaries, particularly with TD-OCT where balloon occlusion is required. Moreover, distinguishing different plaque morphology relies on operator experience and is subject to an element of inter-observer variability, analogous to grey-scale with IVUS. ${ }^{35}$
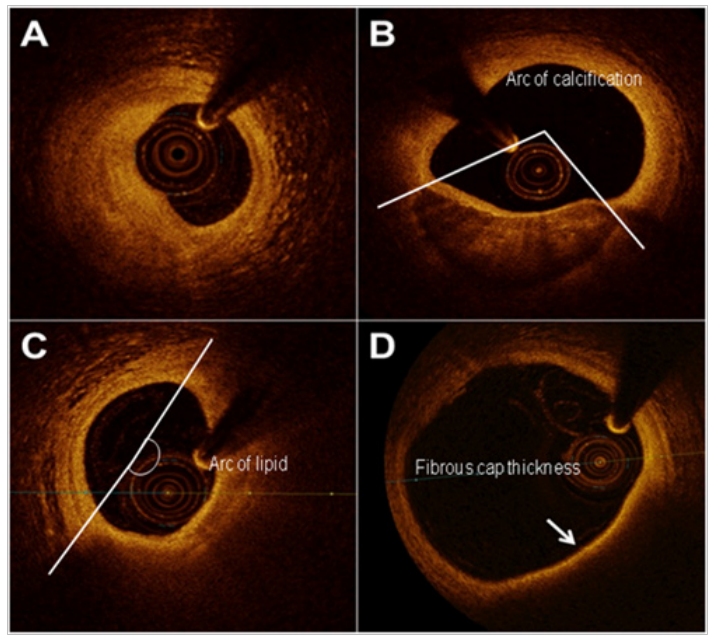

Figure I Frequency domain-optical coherence tomography imaging of atherosclerotic arteries showing A) fibrotic plaque, B) fibrocalcific plaque, C) lipid-rich fibroatheroma and D) a thin-cap (arrow) fibroatheroma. Lipid/ calcification arcs and fibrous cap thickness are marked.

Table I Image characteristics of optical coherence tomography with different plaque morphologies

\section{Tissue characteristics OCT characteristics}

\begin{tabular}{ll}
\hline \multirow{2}{*}{ Fibrous } & Homogenous \\
& High reflectivity \\
& Low attenuation \\
& Diffuse edges \\
Lipid & High reflectivity \\
& High attenuation \\
& Sharp well defined edges \\
Calcific & Low reflectivity (compared to IVUS) \\
& Low attenuation \\
& Mass protruding into vessel lumen \\
Red thrombus & Medium reflectivity \\
& High attenuation \\
& Luminal protrusion \\
White thrombus & Medium reflectivity \\
& Low attenuation \\
High reflectivity \\
High attenuation \\
Low reflectivity (if residual polymer present) \\
Letallic & Low attenuation \\
\hline
\end{tabular}

(IVUS - intravascular ultrasound) 


\section{Conclusion}

Intracoronary OCT currently provides unparalleled high resolution anatomical data which the interventional cardiologist can use to optimise treatment. It allows detailed structural analysis of atherosclerotic plaques and helps characterise the vascular healing process post PCI. However, there are still limitations with this technique and alternative imaging modalities such as IVUS still have a role in plaque delineation and PCI optimisation. Future developments such as micro-OCT will offer spatial resolution that is ten times that of FDOCT, permitting sub-cellular analysis of the atherosclerotic process and improved diagnostic capabilities for the prospective prevention of coronary artery disease. Potential research combining doppler-like signal detection with OCT will allow integration of physiological and anatomical assessment using a single device.

\section{Acknowledgements}

NS is the primary author. PH contributed to the manuscript and MM proof-read the manuscript and is the guarantor.

\section{Conflict of interest}

Author declares that there is no conflict of interest.

\section{References}

1. Global status report on non-communicable diseases 2014. Geneva: World Health Organization; 2015.

2. Ross R. The pathogenesis of atherosclerosis: a perspective for the $1990 \mathrm{~s}$ Nature. 1993;362(6423):801-809.

3. Bezerra HG, Costa MA, Guagliumi G, et al. Intracoronary Optical Coherence Tomography: A Comprehensive Review: Clinical and Research Applications. JACC Cardiovasc Interv. 2009;2(11):1035-1046.

4. Takarada S, Imanishi T, Liu Y, et al. Advantage of next-generation frequency-domain optical coherence tomography compared with conventional time-domain system in the assessment of coronary lesion. Catheter Cardiovasc Interv. 2010;75(2):202-206.

5. Yamagushi M, Terashima M, Akasaka T, et al. Safety and feasibility of an intravascular optical coherence tomography image wire system in the clinical setting. Am J Cardiol. 2008;101(5):562-567.

6. Jang IK, Bouma BE, Kang DH, et al. Visualization of coronary atherosclerotic plaques in patients using optical coherence tomography: comparison with intravascular ultrasound. $\mathrm{J} \mathrm{Am}$ Coll Cardiol. 2002;39(4):604-609.

7. Kawase Y, Hoshino K, Yoneyama R, et al. In vivo volumetric analysis of coronary stent using optical coherence tomography with a novel balloon occlusion-flushing catheter: a comparison with intravascular ultrasound. Ultrasound Med Biol. 2005;31(10):1343-1349.

8. Prati F, RegarE, Mintz GS, et al. Expert review document on methodology, terminology, and clinical applications of optical coherence tomography: physical principles, methodology of image acquisition, and clinical application for assessment of coronary arteries and atherosclerosis. Eur Heart J. 2010;31(4):401-415.

9. McCabe JM, Croce KJ. Optical coherence tomography. Circulation. 2012;126(17):2140-2143.

10. Imola F, Mallus MT, Ramazzotti V, et al. Safety and feasibility of frequency domain optical coherence tomography to guide decision making in percutaneous coronary intervention. Euro Intervention. 2010;6(5):575-581.

11. Barlis P, Gonzalo N, Di Mario C, et al. A multicentre evaluation of the safety of intracoronary optical coherence tomography. Euro Intervention. 2009;5(1):90-95.
12. Gonzalo N, Barlis P, Serruys PW, et al. Incomplete stent apposition and delayed tissue coverage are more frequent in drug-eluting stents implanted during primary percutaneous coronary intervention for STsegment elevation myocardial infarction than in drug-eluting stents implanted for stable/unstable angina: Insights from optical coherence tomography. JACC Cardiovasc Interv. 2009;2(5):445-452.

13. Gonzalo N, Serruys PW, Okamura T, et al. Optical coherence tomography assessment of the acute effects of stent implantation on the vessel wall: A systematic quantitative approach. Heart. 2009;95(23):1913-1919.

14. Yabushita H, Bouma B, Houser S, et al. Characterization of human atherosclerosis by optical coherence tomography. Circulation. 2002;106(13):1640-1645.

15. Schaar JA, Muller JE, Falk E, et al. Terminology for high-risk and vulnerable coronary artery plaques. Report of a meeting on the vulnerable plaque. Eur Heart J. 2004;25(12):1077-1082.

16. Miyamoto Y, Okura H, Kume T, et al. Plaque characteristics of thin-cap fibroatheroma evaluated by OCT and IVUS. JACC Cardiovasc Imaging. 2011;4(6):638-646.

17. Stone GW, Maehara A, Lansky AJ, et al. A prospective natural-history study of coronary atherosclerosis. N Engl J Med. 2011;364(3):226-235.

18. Calvert PA, Obaid DR, O'Sullivan M, et al. Association between IVUS findings and adverse outcomes in patients with coronary artery disease: the VIVA (VH-IVUS in Vulnerable Atherosclerosis) study. JACC Cardiovasc Imaging. 2011;4(8):894-901.

19. Kubo T, Imanishi T, Takarada S, et al. Assessment of culprit lesion morphology in acute myocardial infarction: ability of optical coherence tomography compared with intravascular ultrasound and coronary angioscopy. J Am Coll Cardiol. 2007;50(10):933-939.

20. Kume T, Akasaka T, Kawamoto T, et al. Measurement of the thickness of the fibrous cap by optical coherence tomography. Am Heart J. 2006;152(4):755e1-4

21. Kume T, Okura H, Kawamoto $\mathrm{T}$, et al. Relationship between coronary remodeling and plaque characterization in patients without clinical evidence of coronary artery disease. Atherosclerosis. 2008;197(2):799-805

22. Kubo T, Imanishi T, Kashiwagi M, et al. Multiple coronary lesion instability in patients with acute myocardial infarction as determined by optical coherence tomography. Am J Cardiol. 2010;105(3):318-322.

23. Takarada S, Imanishi $\mathrm{T}$, Kubo $\mathrm{T}$, et al. Effect of statin therapy on coronary fibrous-cap thickness in patients with acute coronary syndrome: assessment by optical coherence tomography study. Atherosclerosis. 2009;202(2):491-497.

24. Kataoka Y, Puri R, Hammadah M, et al. Frequency-domain optical coherence tomographic analysis of plaque microstructures at non-culprit narrowings in patients receiving potent statin therapy. Am J Cardiol. 2014;114(4):549-554

25. Komukai K, Kubo T, Kitabata H, et al. Effect of atorvastatin therapy on fibrous cap thickness in coronary atherosclerotic plaque as assessed by optical coherence tomography: the EASY-FIT study. J Am Coll Cardiol. 2014;64(21):2207-2217.

26. Kume T, Akasaka T, Kawamoto T, et al. Assessment of coronary arterial thrombus by optical coherence tomography. Am J Cardiol. 2006;97:1713-1717.

27. Terashima M, Rathore S, Suzuki Y, et al. Accuracy and reproducibility of stent-strut thickness determined by optical coherence tomography. $J$ Invasive Cardiol. 2009;21(11):602-605.

28. Bouma BE, Tearney GJ, Yabushita H, et al. Evaluation of intracoronary stenting by intravascular optical coherence tomography. Heart. 2003;89(3):317-320 
29. Bezerra HG, Attizzani GF, Sirbu V, et al. Optical coherence tomography versus intravascular ultrasound to evaluate coronary artery disease and percutaneous coronary intervention. JACC Cardiovasc Interv. 2013;6(3):228-236.

30. Prati F, Di Vito L, Biondi-Zoccai G, et al. Angiography alone versus angiography plus optical coherence tomography to guide decisionmaking during percutaneous coronary intervention: the Centro per la Lotta contro l'Infarto-Optimisation of Percutaneous Coronary Intervention (CLI-OPCI) study. EuroIntervention. 2012;8(7):823-829.

31. Guagliumi G, Costa MA, Sirbu V, et al. Strut coverage and late malapposition with paclitaxel-eluting stents compared with bare metal stents in acute myocardial infarction: optical coherence tomography substudy of the Harmonizing Outcomes with Revascularization and Stents in Acute Myocardial Infarction (HORIZONS-AMI) Trial. Circulation. 2011;123(3):274-281
32. Guagliumi G, Musumeci G, Sirbu V, et al. Optical coherence tomography assessment of in vivo vascular response after implantation of overlapping bare-metal and drug-eluting stents. JACC Cardiovasc Interv. 2010;3(5):531-539.

33. Kang SJ, Mintz GS, Akasaka T, et al. Optical coherence tomographic analysis of in-stent neoatherosclerosis after drug-eluting stent implantation. Circulation. 2011;123(25):2954-2963.

34. Manfrini O, Mont E, Leone O, et al. Sources of error and interpretation of plaque morphology by optical coherence tomography. Am J Cardiol. 2006;98(2):156-159. 